\title{
EPICS device support module as ATCA system manager for the ITER fast plant system controller
}

\author{
Paulo F. Carvalho ${ }^{\mathrm{a}, *}$, Bruno Santos ${ }^{\mathrm{a}}$, Bruno Gonçalves ${ }^{\mathrm{a}}$, Bernardo B. Carvalho ${ }^{\mathrm{a}}$, Jorge Sousa ${ }^{\mathrm{a}}$, \\ A.P. Rodrigues ${ }^{a}$, António J.N. Batista ${ }^{a}$, Miguel Correia ${ }^{a}$, Álvaro Combo ${ }^{a}$, Carlos M.B.A. Correia ${ }^{b}$, \\ Carlos A.F. Varandas ${ }^{\mathrm{a}}$ \\ a Associação EURATOM/IST, Instituto de Plasmas e Fusão Nuclear, Instituto Superior Técnico - Universidade Técnica de Lisboa, Lisboa, Portugal \\ b Centro de Instrumentação, Departamento de Física, Universidade de Coimbra, Coimbra, Portugal
}

\section{H I G H L I G H T S}

- In Nuclear Fusion, demanding security and high-availability requirements call for redundancy to be available.

- ATCA based Nuclear Fusion Systems are composed by several electronic and mechanical component.

- Control and monitoring of ATCA electronic systems are recommended.

- ITER Fast Plant System Controller Project CODAC system prototype.

- EPICS device support module as External ATCA system manager solution.

\section{A R T I C L E I N F O}

\section{Article history:}

Available online 14 February 2013

\section{Keywords:}

EPICS

Nuclear

Fusion

Control data acquisition

ATCA systems

Software

Shelf manager

\begin{abstract}
A B S T R A C T
This paper presents an Enhanced Physics and Industrial Control System (EPICS) device support module for the International Thermonuclear Experimental Reactor (ITER) Fast Plant System Controller (FPSC) project based in Advanced Telecommunications Computing Architecture (ATCA) specification. The developed EPICS device support module provides an External System Manager (ESM) solution for monitoring and control the ITER FPSC ATCA shelf system and data acquisition boards in order to take proper action and report problems to a control room operator or high level management unit in case of any system failure occurrence. EPICS device support module acts as a Channel Access (CA) server to report problems and publish ATCA system data information to the control room operator, high level management unit or other CA network clients such as Control System Studio Operator Interfaces (CSS OPIs), Best Ever Alarm System Toolkit (BEAST), Best Ever Archive Utility (BEAUTY) or other CA client applications. EPICS device support module communicates with the ATCA Shelf manager (ShM) using HTTP protocol to send and receive commands through POST method in order to get and set system and shelf components properties such as fan speeds measurements, temperatures readings, module status and ATCA boards acquisition and configuration parameters. All system properties, states, commands and parameters are available through the EPICS device support module CA server in EPICS Process Variables (PV) and signals format. ATCA ShM receives the HTTP protocol messages from the EPICS device support module, validates and takes proper action in order to execute and acknowledge the required command operation and respond with an operation success or error return code. Each ATCA board contains inventory data that can be retrieved by the ShM controller to determine if there is enough power available for the payload process, provide manufacturer data information, retrieve board model and serial numbers and check number of available board sensors their types and fields. The ShM can provide this inventory data information to the EPICS device support module if is required. ShM module communicates with the ATCA boards through the Intelligent Platform Management Interface (IPMI) controller via IPMI communication protocol on redundant IPMI bus. This communication protocol is managed by the embedded Intelligent Management Platform Controller (IPMC) in the ATCA board side and by the embedded Shelf Management Controller (ShMC) in the ShM side. Queried inventory data information becomes available in the EPICS device
\end{abstract}

\footnotetext{
* Corresponding author. Tel.: +351 218417696; fax: +351218417819.

E-mail address: pricardofc@ipfn.ist.utl.pt (P.F. Carvalho).
} 
support module upon ShM command response. EPICS device support module can be a valuable and good approach for ATCA ESM aiming the integration in the ITER Control and Data Acquisition (CODAC) core system according the ITER specification.

(C) 2013 Elsevier B.V. All rights reserved.

\section{Introduction}

ATCA based Nuclear Fusion Systems are composed by several electronic and mechanical components such as control and data acquisition boards, shelf and boards sensors, triggered and nontriggered actuators and cooling capabilities.

Control and monitoring of ATCA electronic systems is recommended to provide system status and sensor health information and to take proper action in case of failure detection, electronic or mechanical components malfunctioning or emergency alarm occurrence.

The next International Nuclear Fusion Reactor, ITER, will require such monitoring and controlling features and capabilities in all of their Control and Data Acquisition Systems.

The ITER Fast Plant System Controller (FPSC) [1] project is a CODAC system prototype proposal that will contribute to the ITER Plant Control Design Handbook (PCDH) [2] effort of standardization, specifically regarding Fast Controller characteristics.

The diagnostic instrumentation and control requirements must comply with standards and guidelines documented in the ITER PCDH and its supporting documents and ITER diagnostic design requirements.

The ITER FPSC specification will be applicable to several diagnostic systems targeting plasma control.

This paper presents an EPICS device support module to serve as External ATCA system manager solution for the ITER (FPSC) [1] project aiming to implement and achieve ATCA shelf system sensors monitoring and actuators control capabilities which will be compliant with ITER requirements and specifications.

\subsection{System overview}

Fig. 1, depicts an overview of ATCA system architecture displaying the main components interconnection and interaction aiming to demonstrate how to access ATCA technology in scope of ITER fast controllers.
- Mini-CODAC (MC) contains all Control System Studio (CSS) operator monitoring and controlling interfaces for system interaction, monitoring and operation.

- Plant System Host (PSH) contains all EPICS Input/Output Controllers (IOCs) and EPICS device support modules for communication with Shelf Management units, Fast Plant Controllers (PCFs) and MCs computers.

- PCF provides ATCA shelf, data acquisition and time switch boards local monitoring and control. Contains required hardware device drivers and EPICS device support modules to configure, initiate, terminate and transfer acquisition data.

- ATCA shelf manager (ShM) module provides an HTTP server to provide network access which allows remote monitoring, operation and control.

\section{Software environment}

Software modules were organized in 3 main blocks according to the ATCA system architecture (Fig. 2).

○ PCF computer which contains RedHat v6.0 operating system with real time kernel capabilities, all software modules, and ITER CODAC system packages as depicted in Table 1.

- ShM module (ADLINK aCM2000 model) contains the embedded controller responsible for all sensors and actuators database information and communication procedures to respond to client commands requests in a HTTP Protocol POST method functions approach. The ShM environment resources capabilities are displayed in Table 2.

- MC remote computer acts as a client of the PCF computer and contains RedHat v6.0 operating system and all EPICS software packages as displayed in Table 3. CSS compliant operator interfaces were developed and deployed in the MC machine to provide local and/or remote sensor monitoring, acquisition parameters configuration and acquisition start/stop operations, actuators control when required, alarm handling and

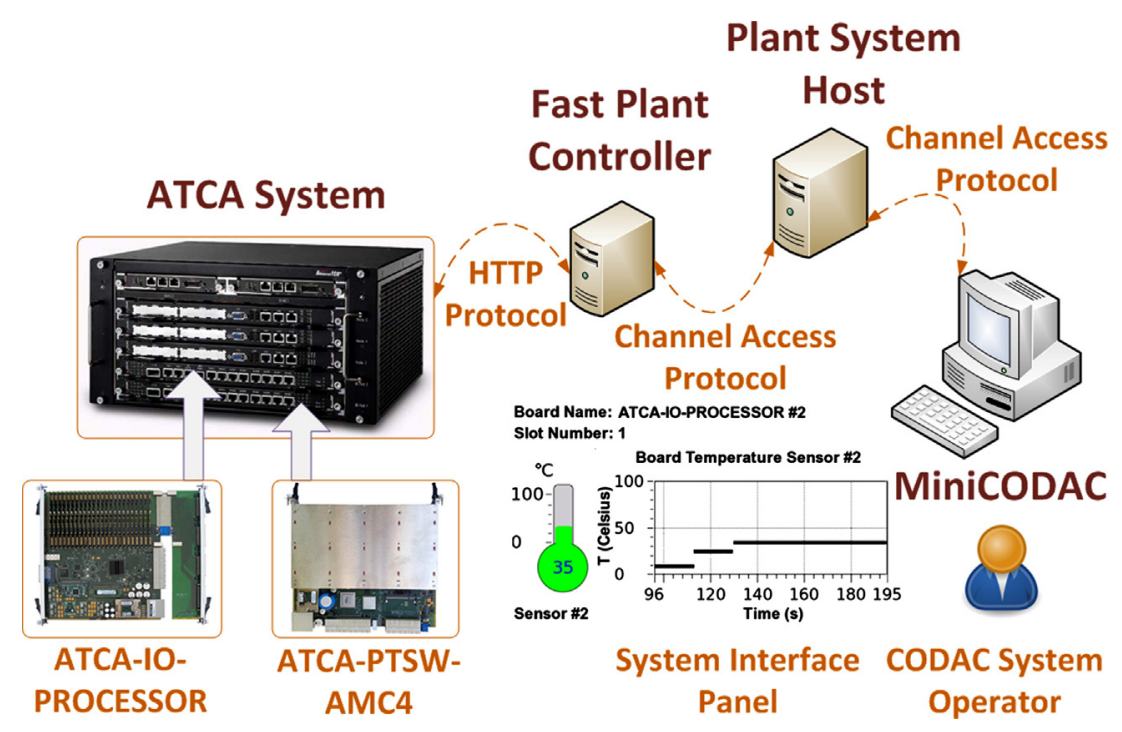

Fig. 1. ITER Fast Plant Controller ATCA system architecture design. 
Table 1

Fast Plant Controller software environment.

\begin{tabular}{|c|c|c|c|}
\hline Component type & Description & Manufacturer & Version \\
\hline Software & CODAC Core System with Real Time kernel capability & ITER & 3.1 \\
\hline Software & ATCA-PTSW-AMC4 Linux device driver & IPFN/IST & 1.0 \\
\hline Hardware & ATCA-PTSW-AMC4 DAQ device & IPFN/IST & 2.1 \\
\hline Software & ATCA-IO-PROCESSOR Linux device driver & IPFN/IST & 1.0 \\
\hline Hardware & ATCA-IO-PROCESSOR DAQ device & IPFN/IST & 2.1 \\
\hline Software & ATCA-PTSW-AMC4 EPICS device support module & IPFN/IST & 1.0 \\
\hline
\end{tabular}

acknowledge, archived and/or non-archived data browsing for visualization.

\section{Implementation}

- MC machine was prepared with ITER CODAC and all EPICS framework packages.

- PCF machine was prepared with ITER CODAC core system using a kernel with real time capabilities and all required EPICS framework tools.

- ATCA data acquisition and timing switch boards installed into the system and correspondent device drivers were also installed via Red-Hat Package Modules (RPMs) previously created packages compliant with ITER CODAC core system rules [3]. EPICS device support external system manager module was also installed in the system via developed RPM package.

- Two ShM units (for redundancy purposes) installed in the ATCA system crate and Ethernet settings were properly configured to provide network access. EPICS device support system manager to ATCA ShM communication architecture is depicted in Fig. 3.

The HTTP protocol implemented to communicate with ShM HTTP server is based in a send/receive functions library using the TCP/IP POST method to send HTTP commands. POST method was used instead of GET method for:

- Security purposes: GET requests can be seen in the URL HTTP page while POST requests are encapsulated in the body of the HTTP request.

- Type of data used: GET requests are text-only based while POST requests can be either text or binary data.

- Data set required: GET requests are restricted to use ASCII characters while POST requests use an attribute with a value.

POST commands sent to the HTTP server are received, interpreted, executed and corresponded response messages sent back

Table 2

Shelf manager unit resources.

\begin{tabular}{ll}
\hline CPU & Type $=$ e300 Core Clock $=249 \mathrm{MHz}$ \\
\hline Memory A & $128 \mathrm{MB}$ SDRAM host memory \\
Memory B & $64 \mathrm{MB}$ flash memory \\
Operating System & Linux SHMM 1500 \\
& Kernel version 2.4 .25 Power PC \\
\hline
\end{tabular}

Table 3

Mini-CODAC software environment.

\begin{tabular}{llll}
\hline Component type & Description & Manufacturer & Version \\
\hline Software & CODAC Core System & ITER & 3.1 \\
Software & Control System Studio OPI IDE & ITER & 3.1 \\
Software & BEAST Alarm Handler & ITER & 3.1 \\
Software & BEAUTY Archive Engine & ITER & 3.1 \\
Software & EPICS framework required & ITER & 3.1 \\
& software packages & & \\
\hline
\end{tabular}

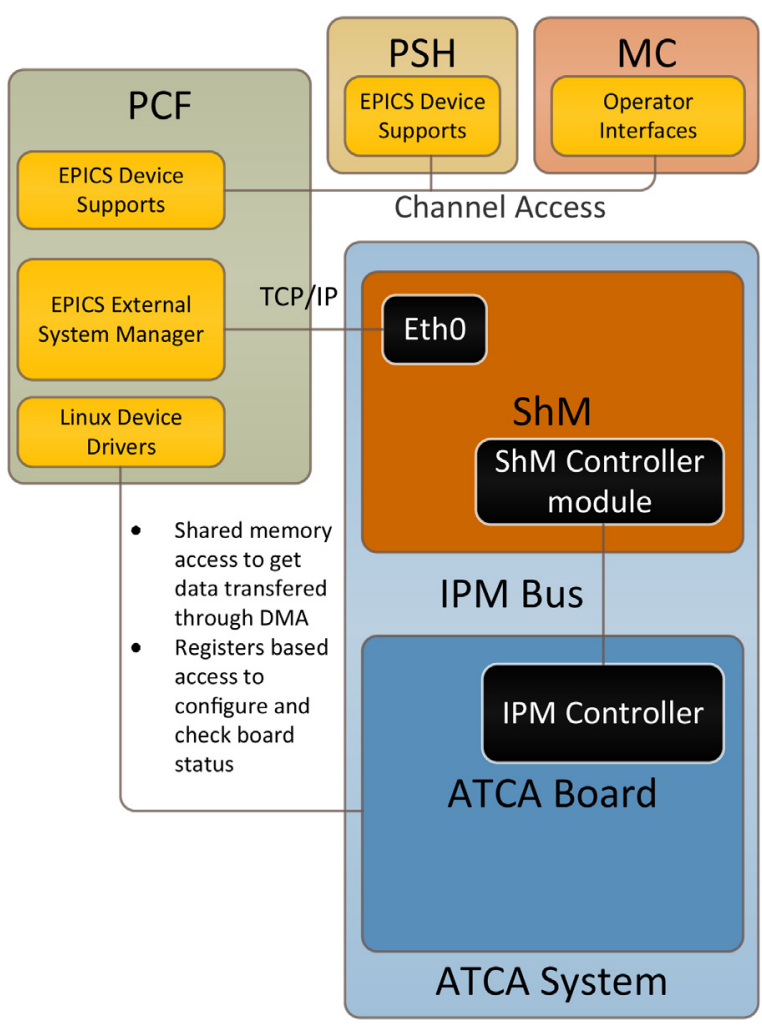

Fig. 2. Software modules block diagram.

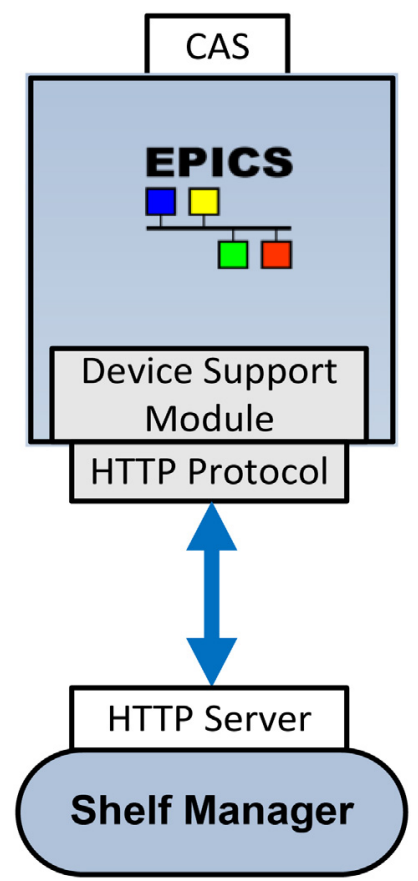

Fig. 3. EPICS device support module o ATCA ShM block diagram. 


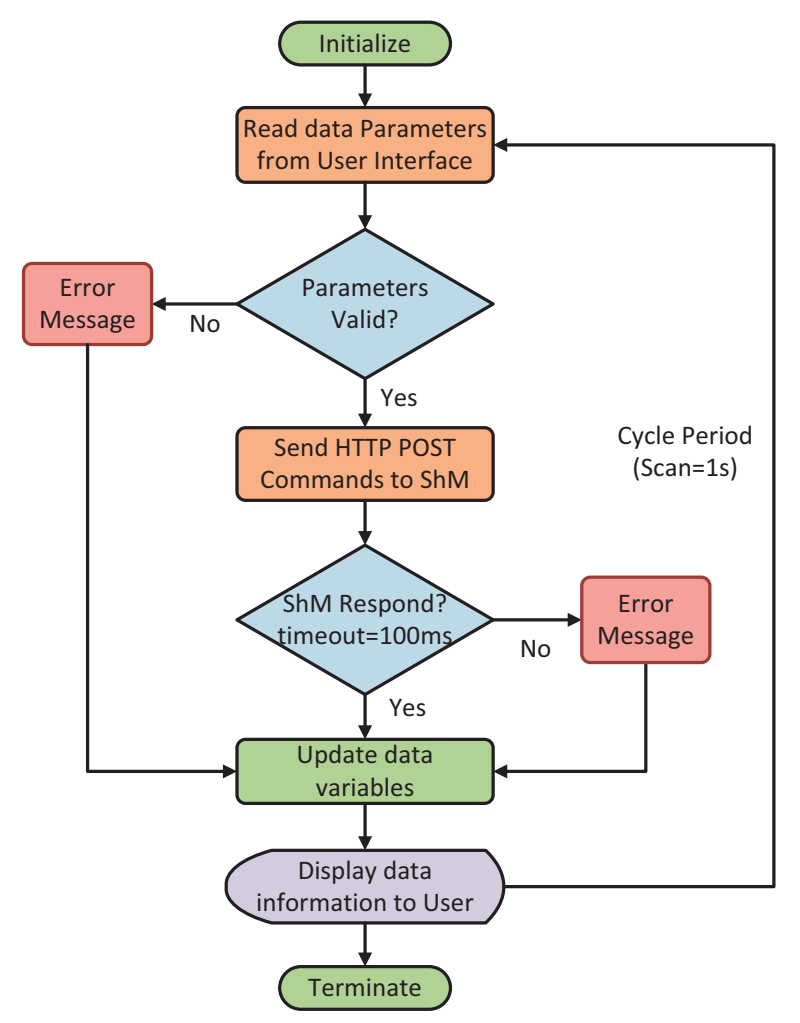

Fig. 4. EPICS device support module to ATCA ShM communication protocol flowchart.

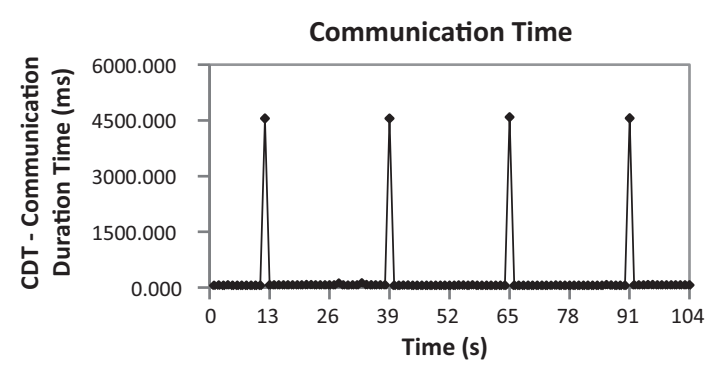

Fig. 5. CDT of 1 sensor data requests.

to the EPICS device support module which parses the required information and updates PVs values in accordance. The HTTP Communication Protocol flowchart algorithm developed and implemented is depicted in Fig. 4.

Sensors to be monitored or controlled are selected by the ITER control room operator via operator interfaces. This information is passed to the EPICS device support and translated into a sensor address id parameters and values format that needs to pass through a validation process. If passed validation process then data are sent by the EPICS device support to the ShM unit via HTTP protocol in a periodic time of $1 \mathrm{~s}$ per senor. If ShM responds within a timeout of $10 \mathrm{~s}$ then the device support receives and updates PVs values and sends updated PVs information to the operator interfaces for data visualization.

\section{Results}

Fig. 5 shows the Communication Duration Time (CDT) when only one sensor is read from the shelf manager. We observed a particularly $25 \mathrm{~s}$ periodic event.

The CDT obtained for the detected event was 4563.103 ms (average of the high value 4 points observed in Fig. 5). Analyzing deeply

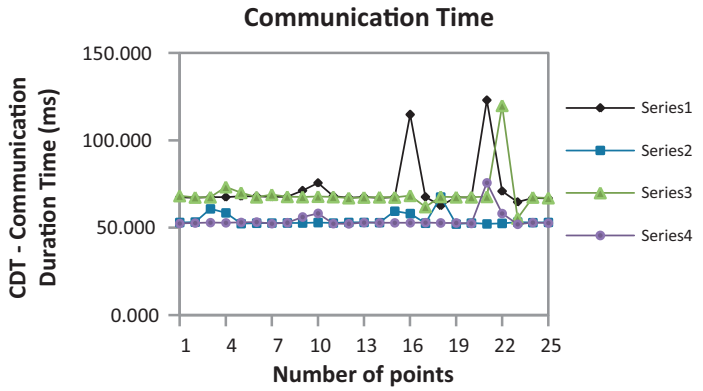

Fig. 6. CDT of 1 sensor requests without the interrupt event duration time in a 4 data windows with 25 points each.

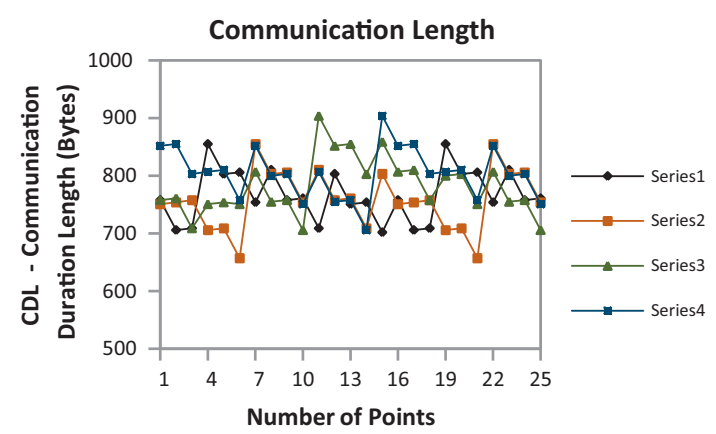

Fig. 7. CDL of 1 sensor data requests without the interrupt event data length in a 4 data windows with 25 points each.

the ShM unit running processes it was found an interrupt event which could be the cause of this event. A possible explanation for this interrupt can be related with a communication message buffer flush operation that prepares the HTTP communication buffer for new data packets. To study the impact of the detected interrupt event in the duration of the communication protocol we decide for a two step approach.

- Step 1: calculating the CDT and Communication Data Length (CDL) average values using a 25 point time in 4 windows without the interrupt event occurrence time as displayed in Fig. 1.

- Step 2: shifting by one point the data sample points in order to add the event time duration point, calculate the CDT and CDL average value (Figs. 6 and 7).

$\frac{\sum_{k=1}^{k=4}\left(\sum_{i=1}^{i=25} \mathrm{CDT}(i)\right) / 25}{4}=63.104 \mathrm{~ms}$

$\frac{\sum_{k=1}^{k=4}\left(\sum_{j=1}^{j=25} \operatorname{CDT}(j)\right) / 25}{4}=797$ bytes

$\frac{\sum_{k=1}^{k=4}\left(\sum_{z=1}^{z=25} \operatorname{CDT}(z)\right) / 25}{4}=241.971 \mathrm{~ms}$

The calculated average value of the communication process obtained without the event time was $63.104 \mathrm{~ms}$, Eq. (1), for a calculated average data length of 797 bytes, Eq. (2). The event impact factor can be calculated by a ratio between Eqs. (3) and (1) results, $241.971 \mathrm{~ms} / 63.104 \mathrm{~ms}=3.834$.

Note that each time point value was requested by the EPICS device support module to the ATCA ShM with a $1 \mathrm{~s}$ periodicity clock window.

From instant $t=104 \mathrm{~s}$ ( $25 \mathrm{~s}$ times 4 data windows added to 4 peak interrupt event points, starting at $1 \mathrm{~s}) 182$ sensors data information began to be requested by the EPICS device support to the ATCA ShM with the same a $1 \mathrm{~s}$ periodicity clock window. Monitoring the same 


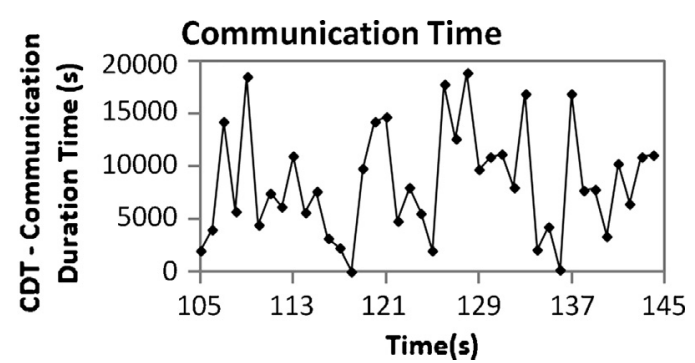

Fig. 8. CDT of 1 sensor request in the presence of all 182 system sensors data information requests.

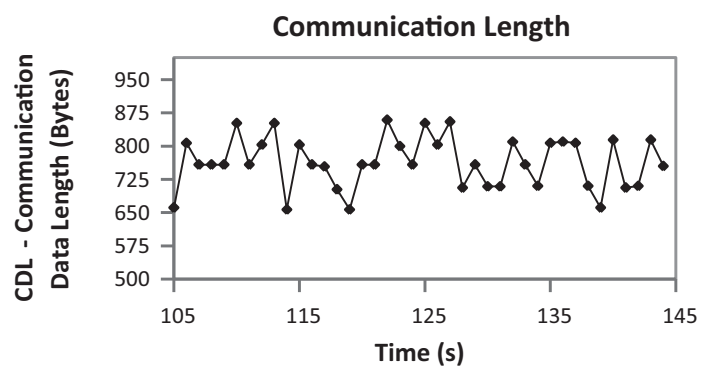

Fig. 9. CDL for 1 sensor request in the presence of all 182 system sensors requests.

previous sensor duration time we obtain the behavior depicted in Fig. 8.

Observing Fig. 8 it is clearly that the ShM response to the EPICS device support requests communication behavior has changed. Took more time for the ShM module to respond (average $=8483.708 \mathrm{~ms}$, Fig. 8) to transfer almost the same amount of CDL, average $=763.375$ bytes, Fig. 9 .

The interrupt event is still present but embedded in the communication duration time points.

\section{Conclusions}

Throughout the development we found that the limitations were twofold: (i) EPICS device support approach to monitor several sensors at a high frequency rate once EPICS framework is better for slow monitoring and control experiences; and (ii) increase of number of sensors leads to low performance in HTTP communication as described in previous sections.

In spite of these limitations this implementation has several advantages: (i) EPICS device support implementation compliant with ITER specifications; (ii) local or remote slow sensor monitoring and actuator control with capability to detect, notify a control room operator and acknowledge possible alarm occurrences; and (iii) highly configurable and adaptable to different shelf management units and to new shelf and board sensors implementations.

In our opinion, to aim better performance, it should be possible to: (a) replace the ShM processor or the entire ShM unit for a better one (depending of the shelf model used); (b) replace or improve the HTTP communication protocol implemented, if possible; or (c) implement a sensor/actuator dedicated server in the ShM unit to provide better communication performance to connected clients and better sensor filtering functions in order to fetch only data of some specific sensors (not all) at a specific sensor update rate.

Once the results presented were obtained using the standard Linux API commands to monitor system performance, future work will require to implement and to perform further tests based in specification documents and ITER not yet developed tools for the above solutions and others that may possible occur to verify communication performance improvements.

\section{Acknowledgments}

This work has been sponsored by the Contract of Association between European Atomic Energy Community and Instituto Superior Técnico (IST) and by the Contract of Associated Laboratory between Fundação para a Ciência e Tecnologia (FCT) and IST.

The content of publication is the sole responsibility of the authors and it does not necessarily represent the views of the Commission of the European Union or FCT.

\section{References}

[1] B. Gonçalves et al., ITER fast plant system controller prototype based on ATCA Platform, Fusion Engineeering and Design, in press

[2] CODAC Team, Plant Control Design Handbook (ITER D 27LH2V v6.1), 2011.

[3] CODAC Core System Application Development Manual (ITER_D_33T8LW). 